\title{
Validez estructural y convergente de la escala de engrandecimiento marital en México
}

\section{Structural and convergent validity of the Marital Aggrandizement Scale (MAS) in Mexico}

José Moral de la Rubia

Universidad Autónoma de Nuevo León (UANL)

\section{Resumen}

La tendencia a exagerar las cualidades positivas de la pareja e ignorar sus defectos se denomina engrandecimiento, representa una variable importante para el estudio de la pareja y existe una escala para su evaluación. Esta escala ha sido validada en México, con base en el uso de un muestreo no probabilístico e ignorando el nivel de medida ordinal de sus ítems. Este estudio tiene como objetivos estimar la consistencia interna de la escala de engrandecimiento marital (Marital Aggrandizement Scale, MAS, por sus siglas en inglés), validar un modelo unidimensional y mostrar la validez convergente de la MAS. Se administró la MAS y la escala de valoración de la relación (Relationship Assesment Scale, RAS, por sus siglas en inglés), a 807 personas casadas o en unión libre de Monterrey, México, a partir de un muestreo de rutas aleatorias. Tres de los cuatro ítems inversos y un ítem directo presentaron problemas de consistencia interna y validez. Con la eliminación de estos cuatro ítems (MAS_14), el número de factores en la matriz de correlaciones policóricas fue uno por el criterio de Velicer. El ajuste del modelo unidimensional con sus residuos independientes fue aceptable por mínimos cuadrados no ponderados $(\chi 2 / \mathrm{gl}=4.69$, AGFI $=.97, \mathrm{NFI}=.96$ y SRMR $=.07)$. La consistencia interna de los catorce ítems fue buena ( $\alpha$ ordinal $=.87$ ). La correlación entre MAS_14 y RAS fue alta $(r=.65)$. Se concluyó, por lo tanto, que la MAS reducida a catorce ítems es consistente, se validó el modelo unidimensional, la MAS presentó validez convergente con la satisfacción con la relación, de ahí que el engrandecimiento marital y la satisfacción con la relación dos conceptos distinguibles.

Palabras clave: engrandecimiento, satisfacción con la relación, pareja, psicometría, México.

Nota del autor

José Moral de la Rubia, Facultad de Psicología, Universidad Autónoma de Nuevo León (UANL).

La correspondencia en relación con este artículo debe dirigirse a José Moral de la Rubia, Facultad de Psicología, UANL, calle Dr. Carlos Canseco \#110, colonia Mitras Centro, C.P. 64460, Monterrey, Nuevo León, México.

Dirección electrónica: jose_moral@hotmail.com 


\begin{abstract}
The tendency to exaggerate the positive qualities of the intimate partner and ignore its flaws is named aggrandizement, represents an important variable for the study of the couple, and there exists a scale for its assessment. This scale has been validated in Mexico, using a non-probability sampling and ignoring the ordinal level of measurement of its items. The aims of this study were to estimate the internal consistency of the Marital Aggrandizement Scale (MAS), validate a single-factor model, and show the convergent validity of the MAS. The MAS and the Relationship Assessment Scale (RAS) were applied to 807 married or cohabiting persons from Monterrey, Mexico, using a random-path sampling. Three of the four inversely keyed items and a positively keyed item had problems of internal consistency and validity. With the elimination of these four items (MAS_14), the number of factors in the matrix of polychoric correlations was one by the Velicer's criterion. The fit of the one-factor model with all its independent measurement residuals was acceptable by the method of Unweighted Least Squares $(\chi 2 / \mathrm{df}=4.69, \mathrm{AGFI}=.97, \mathrm{NFI}=.96$, and SRMR $=.07)$. Internal consistency of the 14 items was good (ordinal $\alpha=.87$ ). The correlation between MAS_14 and RAS was high $(r=.65)$. It is concluded that the MAS reduced to 14 items is consistent, the one-factor model is validated, the MAS shows convergent validity with relationship satisfaction, and marital aggrandizement and relationship satisfaction are two distinguishable concepts.
\end{abstract}

Keywords: Aggrandizement, relationship satisfaction, partner, psychometrics, Mexico.

El engrandecimiento marital se define como un estilo de respuesta que conduce a reportar una imagen exageradamente positiva del cónyuge y la relación marital, y se concibe como un rasgo funcional (O'Rourke, \& Cappeliez, 2002).

Las escalas de medición de la satisfacción con la relación de pareja se suelen hallar influenciadas por una tendencia a idealizar a la pareja y a dar respuestas distorsionadas en un sentido socialmente deseable, proporcionando la imagen de una relación feliz y en perfecta armonía, por lo cual se recomienda controlar la tendencia a engrandecer la relación de pareja (West, Dovidio, \& Pearson, 2014). Para controlar este sesgo, en lugar de escalas de aplicación general (a cualquier situación social), O’Rourke y Cappeliez (2002) sugieren emplear escalas específicas para la situación de pareja. A tal fin, estos investigadores canadienses desarrollaron la MAS.

La MAS en su versión original se escribió en inglés; quedó integrada por 18 ítems tipo Likert, con siete opciones de respuesta, y una estructura unidimensional con un ajuste aceptable, tras introducir correlaciones entre los residuos de medida (O'Rourke, \& Cappeliez, 2002). Fue traducida al francés por sus autores, según el método de traducción inversa, dando lugar a la Échelle d'Embellissement Conjugal (EEC); en una muestra de personas francófonas, la EEC mostró consistencia interna excelente $(\alpha$ de Cronbach $=.91)$ y alta estabilidad en un periodo de 33 días $(r[15]=.88, p<.001)$ (O’Rourke, \& Cappeliez, 2003). 
La MAS fue traducida al español por Moral (2007), quien usó el método de traducción inversa. En una muestra incidental de 100 parejas mexicanas de población general, Moral (2007) obtuvo una consistencia interna aceptable entre los 18 ítems de la MAS ( $\alpha$ de Cronbach $=.77$ ). El número de factores fue dos por el criterio de Cattell. Para mejorar la consistencia interna $(\alpha$ de Cronbach $=.78)$ y lograr una configuración factorial más definida, Moral (2007) aconsejó eliminar los ítems inversos 13 y 17. Al extraer dos factores por el método de ejes principales (Principal Axis, PA, por sus siglas en inglés), se explicó $28.8 \%$ de la varianza total de los 16 ítems seleccionados. Tras rotar la matriz factorial por el método Oblimín, se configuró un factor de sentimientos positivos (enamoramiento, comprensión y satisfacción) con doceindicadores (ítems 1, 2, 3, 4, 5, 9, 10, 12, 14, 15, 16 y 18) y consistencia interna aceptable ( $\alpha$ de Cronbach $=.79$ ), además de otro factor de sentimientos y eventos negativos pocos frecuentes (discusión, enojo y defectos) con cuatro indicadores (ítems $6,7,8$ y 11) y consistencia interna cuestionable ( $\alpha$ de Cronbach $=.61)$, la cual no se incrementó con la eliminación de ningún ítem. El ajuste del modelo de dos factores correlacionados por máxima verosimilitud fue aceptable $(\chi 2 / g l=$ 1.865, RMSSR $=.07$, RMSEA $=.07, P G I=.95$, $A P G I=.93, G F I=.89, A G F I=.86$ y $C F I=.85)$, $\mathrm{y}$ su bondad de ajuste fue significativamente mayor al modelo de un factor con 16 indicadores $(\Delta \chi 2[1]=63.11, p<.001) \mathrm{y}$ con 18 indicadores $(\Delta \chi 2[32]=128.50, p<.001)$. La correlación entre ambos factores fue positiva y moderada $(r[200]=.40, p<.001)$.

Las MAS también ha sido traducida al hebreo por O'Rourke, Polchenko, Bachner y Carmel (2012), con base en el método de traducción inversa. Se aplicó la versión en inglés de la MAS a una muestra de 215 adultos mayores canadienses y se administró la versión en hebreo a otra muestra de 206 adultos mayores israelitas. Se contrastó el modelo unidimensional con 18 indicadores, y se concluyeron correlaciones entre los residuos de medida. En la muestra canadiense, el ajuste fue bueno por máxima verosimilitud $(\chi 2 / g l=1.31, C F I=.97, S R M R$ $=.05 \mathrm{y} R M S E A=.04)$ y la consistencia interna alta $(\alpha=.87)$. En la muestra israelita, el ajuste fue aceptable $(\chi 2 / g l=1.91, C F I=.94, S R M R$ $=.06$ y $R M S E A=.07)$ y la consistencia interna excelente $(\alpha=.90)$. Al hacer un análisis multigrupo entre ambas muestras, se evidenció diferencia significativa sólo en los ítems 3 y 17 (O'Rourke et al., 2012).

Desde los estudios revisados, se observa que se han propuesto dos modelos factoriales para la MAS. Uno es el de un factor con correlaciones entre los errores de medida o con reducción de indicadores para lograr un buen ajuste a los datos. El otro es el de dos factores correlacionados. La determinación del número de factores en ambos modelos se basa en el criterio de Cattell. Actualmente, se señala la importancia crítica de determinar el número de factores acudiendo a la convergencia de criterios; cuatro son los más recomendados: el de Horn, coordenadas 
óptimas, datos de comparación y el de Velicer (Courtney, 2013).

Se ha enfatizado que los ítems tipo Likert son variables ordinales y la correlación de Pearson subestima la relación lineal entre estas variables, siendo más adecuada la correlación policórica. Como consecuencia, la determinación del número de factores y su extracción debería realizarse desde la matriz de correlaciones policóricas o de Spearman y no desde la matriz de correlación de Pearson (Gaskin, \& Happell, 2014). Lo mismo se considera con respecto al cálculo de la consistencia interna. El coeficiente alfa de Cronbach subestima la consistencia interna entre variables ordinales frente al coeficiente alfa ordinal. El primero se basa en la media de las correlaciones de Pearson entre los ítems, y el segundo, en la media de las correlaciones policóricas (Gadermann, Guhn, \& Zumbo, 2014).

Al extraer los factores se sugieren métodos que descompongan la varianza de los ítems en varianza compartida y en varianza única. Los estudios con la MAS han aplicado estos métodos en el análisis factorial exploratorio, como máxima verosimilitud (Maximum Likelihood Estimation, ML, por sus siglas en inglés) y PA. Debe señalarse que ML requiere variables numéricas y normalidad multivariada, que son condiciones incumplidas por ítems ordinales; cuando los métodos de mínimos cuadrados no ponderados (Unweighted Least Squares, ULS, por sus siglas en inglés) o libres de escala (Scale-Free Least Squares, SLS, por sus siglas en inglés) no requieren estos supuestos y rinden mejor con ítems ordinales (Valdivieso, 2013).

Se critica el intentar mejorar el ajuste de un modelo al introducir correlaciones entre residuos de medida, ya que se puede estar forzando un modelo mal especificado sin dar cuenta de la interacción subyacente a dichos residuos; por el contrario, se aconseja explorar de forma empírica y/o teórica modelos alternativos (Hayduk, 2014).

Finalmente, cabe señalar que el concepto de engrandecimiento marital, entendido como un sesgo funcional en el reporte de la satisfacción marital, debe diferenciarse de la satisfacción marital y, por consiguiente, la correlación entre una escala que mida engrandecimiento marital $\mathrm{y}$ otra que mida satisfacción marital no puede ser perfecta $(r \geq .90)$, ni muy alta $(r \geq .70)$, esto es, la varianza compartida debería ser menor que la mitad. O'Rourke y Cappeliez (2002) reportaron una correlación entre la MAS y la escala de ajuste diádico (Dyadic Adjustment Scale, DAS, por sus siglas en inglés) de Spanier (1976) alta $(r[350]=.53, p<.001)$. A su vez, Moral (2007) encontró correlaciones altas entre la MAS-16 ( $\sin$ los ítems 13 y 17) y la DAS $(r[200]=.641, p<.001)$, la RAS de Hendrick $(1988)(r[200]=.657, p<.001)$ y la Escala de Satisfacción Marital (ESM) de Pick y Andrade $(1988)(r[200]=.611, p<.001)$. Por tanto, estos estudios con las correlaciones altas y menores que .70 reportadas proporcionan validez de constructo a la MAS. 
Considerando los distintos resultados sobre la estructura factorial de la MAS y los métodos recomendados para variables en una escala de medida ordinal, el presente estudio, realizado con población de personas casadas o en unión libre de la ciudad de Monterrey en México, tiene como objetivos: 1) describir las distribuciones de los 18 ítems de la MAS y estudiar sus propiedades de discriminación, consistencia interna y validez convergente (con una escala de satisfacción con la relación de pareja); 2) estimar la consistencia interna entre sus 18 ítems; 3) determinar el número de factores por la convergencia de criterios; 4) explorar los factores en caso de que sean dos o más, y calcular la consistencia interna de estos factores; 5) contrastar el ajuste del modelo de un factor y posibles modelos alternativos; y 6) comprobar la validez convergente de la MAS con respecto a la escala de satisfacción con la relación de pareja.

Se tiene como expectativas que: 1) los ítems sean discriminativos, consistentes $y$ estén asociados con la escala de satisfacción con la relación, pudiendo los ítems 13 y 17 tener problemas de consistencia interna; 2) una consistencia interna alta o excelente en la escala; 3) que el número de factores sea uno; 4) en caso de ser dos, se espera un factor de sentimientos positivos y otro de sentimientos y eventos negativos pocos frecuentes, siendo la consistencia interna del primero alta y la del segundo aceptable; 5) una estructura de un factor con buen ajuste, una vez especificadas correlaciones entre sus residuos o una vez reducido el número de indicadores, pudiendo la estructura de dos factores correlacionados tener un ajuste mejor; y 6) correlación alta entre la MAS y la escala de satisfacción con la relación de pareja, con una varianza compartida menor que la mitad, al estarse evaluando dos conceptos distinguibles.

\section{Método}

\section{Participantes}

Los criterios de inclusión fueron: tener al menos 18 años, estar casado o vivir en unión libre con una pareja del sexo opuesto, residir en la ciudad de Monterrey en México, saber leer y escribir y dar el consentimiento informado. Los criterios de eliminación consistieron en: cuestionario incompleto e informar que la pareja estuvo presente al momento de contestar el instrumento, leyendo o preguntando por las respuestas.

Se empleó un muestreo de rutas aleatorias, seleccionándose de forma aleatoria 81 direcciones a partir de la Guía Roji (dos direcciones por encuestador). Cada dirección se tomó como punto de partida de un itinerario. En cada itinerario se recolectaron diez unidades (con consentimiento informado y datos completos). Se pretendía que fueran cinco mujeres y cinco hombres independientes (uno por casa) en cada itinerario. El encuestador tocaba el timbre o puerta de la casa, si ésta era abierta, se presentaba el entrevistador 
como un ayudante de investigación, se informaba de los objetivos del estudio (estudio sobre dinámica de la vida marital o de convivencia por medio de un cuestionario), del responsable del mismo y criterios de inclusión. Si la persona deseaba participar voluntariamente y satisfacía los criterios de inclusión, se dejaba un cuestionario para recogerlo media hora después o cuando lo indicase la persona. Se indicaba que debía contestarlo a solas, sin que su pareja leyese las preguntas o respuestas. Al recogerlo se preguntaba si la pareja había estado presente a la hora de contestar o había visto sus respuestas. En caso afirmativo, el cuestionario no se incluía en la base de datos. En caso contrario, se revisaba que estuviera resuelto en su totalidad; si había preguntas vacías, se pedía amablemente que se respondiesen. Las colonias por las que discurrían las rutas seleccionadas fueron Mitras Norte, Mitras Centro, Mitras Sur y La Coyotera, ya que con estas áreas se conseguía abarcar todos los estratos socioeconómicos sin alejarse en exceso de la facultad, ubicada en Mitras Centro.

Los entrevistadores fueron alumnos de primer semestre de la Facultad de Psicología de la Universidad Autónoma de Nuevo León (UANL) que cursaban la materia de Investigación en Psicología. Recibieron entrenamiento por parte del responsable del proyecto de investigación para colectar los casos y fueron compensados con puntos extras en su calificación.

Para determinar el tamaño de la muestra se empleó el último censo de población y vivienda (Instituto Nacional de Estadística, Geografía e Informática; INEGI, 2016). Bajo un modelo de curva normal, si se desea estimar la media de la MAS con una desviación estándar esperada de 15.37 en un rango de 1 a 126 (Moral, 2007) y con un error absoluto de estimación de 1.06 $(M=76.52$, IC 95\%: 75.46, 77.58), se requeriría una muestra mínima de 806 individuos, esto es, una fracción de muestreo de $0.2 \%$.

Para tal efecto, se completó una muestra de 807 participantes, de los cuales 431 (53.4\%) fueron mujeres y $376(46.6 \%)$ hombres, sin diferencia de frecuencia estadísticamente significativa entre ambos sexos por la prueba binomial $(p=.057)$. Con respecto al estado civil, $727(90 \%)$ de los 807 participantes indicaron estar casados y $80(10 \%)$ en unión libre. La media de años de vida conjunta fue $11.61(D E=9.33)$, variando de 6 meses a 43 años. De las 807 personas encuestadas, 677 (84\%) dijeron tener hijos y 130 (16\%) no tener hijos. La media de hijos fue $1.99(D E=1.30)$, variando de 0 a 9. La media de edad fue 35.40 años $(D E=10.41)$ y la mediana 34 , variando de 18 a 94 años. La mediana de escolaridad $(M d n=2)$ correspondió a secundaria (de siete a nueve años de escolaridad) y la media ( $M=$ 2.51) quedó entre estudio de secundaria y media superior (en torno a 10 años de escolaridad). La mediana y la media de ingreso mensual de la familia correspondieron al rango de 6,000 a 11,999 pesos (28\%), variando de menor de 3,000 (7.6\%) a más de 92,000 (1.9\%). Con respecto a la religión, 662 de los 807 participantes $(82 \%)$ dijeron ser cristianos católicos; 76 (9.4\%), cristianos no católicos; 13 (1.6\%), pertenecer a otras religiones; y $56(6.9 \%)$, a ninguna (tabla 1$)$. 
Tabla 1

Distribución de frecuencias de las variables sociodemográficas

\begin{tabular}{|c|c|c|c|}
\hline Variable & Valor & $n(p)$ & $N(P)$ \\
\hline \multirow[t]{2}{*}{ Sexo } & Mujer & $431(53.4 \%)$ & \\
\hline & Hombre & $376(46.6 \%)$ & \\
\hline \multirow[t]{5}{*}{ Años de edad } & {$[18,30]$} & $285(35.3 \%)$ & $285(35.3 \%)$ \\
\hline & {$[30,40]$} & $230(28.5 \%)$ & $515(63.8 \%)$ \\
\hline & {$[40,50]$} & $220(27.3 \%)$ & $735(91.1 \%)$ \\
\hline & {$[50,60]$} & $60(7.4 \%)$ & $795(98.5 \%)$ \\
\hline & {$[60,94]$} & $12(1.5 \%)$ & $807(100 \%)$ \\
\hline \multirow[t]{5}{*}{ Escolaridad } & Primaria & $173(21.4 \%)$ & $173(21.4 \%)$ \\
\hline & Secundaria & $231(28.6 \%)$ & $404(50.1 \%)$ \\
\hline & Media superior & $218(27 \%)$ & $622(77.1 \%)$ \\
\hline & Licenciatura & $164(20.3 \%)$ & $786(97.4 \%)$ \\
\hline & Posgrado & $21(2.6 \%)$ & $807(100 \%)$ \\
\hline \multirow[t]{2}{*}{ Casado } & Sí & $727(90.1 \%)$ & \\
\hline & No & $80(9.9 \%)$ & \\
\hline \multirow[t]{6}{*}{ Años de matrimonio o unión libre } & {$[0,7.17]$} & $361(44.7 \%)$ & $361(44.7 \%)$ \\
\hline & {$[7.17,14.34]$} & $146(18.1 \%)$ & $507(62.8 \%)$ \\
\hline & {$[14.34,21.51]$} & $171(21.2 \%)$ & $678(84 \%)$ \\
\hline & {$[21.51,28.67]$} & $91(11.3 \%)$ & $769(95.3 \%)$ \\
\hline & {$[28.67,35.84]$} & $27(3.3 \%)$ & $796(98.6 \%)$ \\
\hline & {$[35.84,43]$} & $11(1.4 \%)$ & $807(100 \%)$ \\
\hline \multirow{7}{*}{$\begin{array}{l}\text { Ingresos económicos familiares al mes } \\
\text { en el último año en pesos mexicanos }\end{array}$} & $<3000$ & $61(7.6 \%)$ & $61(7.6 \%)$ \\
\hline & {$[3,000,6,000]$} & $220(27.3 \%)$ & $281(34.8 \%)$ \\
\hline & {$[6,000,12,000]$} & $226(28.0 \%)$ & $507(62.8 \%)$ \\
\hline & {$[12,000,24,000]$} & $174(21.6 \%)$ & $681(84.4 \%)$ \\
\hline & {$[24,000,48,000]$} & $77(9.5 \%)$ & $758(93.9 \%)$ \\
\hline & {$[48,000,92,000]$} & $34(4.2 \%)$ & $792(98.1 \%)$ \\
\hline & $\geq 92,000$ & $15(1.9 \%)$ & $807(100 \%)$ \\
\hline \multirow[t]{6}{*}{ Número total de hijos } & 0 & $130(16.1 \%)$ & $130(16.1 \%)$ \\
\hline & 1 & $149(18.5 \%)$ & $279(34.6 \%)$ \\
\hline & 2 & $237(29.4 \%)$ & $516(63.9 \%)$ \\
\hline & 3 & $211(26.1 \%)$ & $727(90.1 \%)$ \\
\hline & 4 & $60(7.4 \%)$ & $787(97.5 \%)$ \\
\hline & $\geq 5$ & $20(2.5 \%)$ & $807(100 \%)$ \\
\hline \multirow[t]{4}{*}{ Religión } & Cristiana católica & $662(82.0 \%)$ & \\
\hline & Cristiana no católica & $76(9.4 \%)$ & \\
\hline & Otra & $13(1.6 \%)$ & \\
\hline & Ninguna & $56(6.9 \%)$ & \\
\hline
\end{tabular}

$n=$ frecuencia absoluta simple, $p=$ porcentaje simple,

$N=$ frecuencia absoluta acumulada, $P=$ porcentaje acumulado. 
En la población de Monterrey se han registrado 98 hombres por cada 100 mujeres; una mediana de 30 años de edad que sube a 34 al eliminar $31 \%$ de la población menor de 18 años; una media de 9.5 años de escolaridad (entre primer semestre y segundo de educación media superior); una media de ingresos mensuales de 6,700 pesos mexicanos; una media de dos hijos; y $83 \%$ de la población con afiliación religiosa cristiana católica, $7.4 \%$ cristiana no católica, $2.5 \%$ de otra religión y $6.9 \%$ sin religión (INEGI, 2016). Estos datos poblacionales son estadísticamente equivalentes a los de la muestra, como en la frecuencia de ambos sexos $(\chi 2[1, N=807]=1.87, p=.171)$ y de la afiliación religiosa $(\chi 2[3, N=807]=7.13, p=.068)$ así como en la media de hijos $(t[806]=-0.27, p=$ .787), o muy semejantes, como en la mediana de edad, de escolaridad y de ingresos. Si se reduce la población de la zona metropolitana de Nuevo León a la de adultos casados y en unión libre, se tendría $89 \%$ casados y $11 \%$ en unión libre, esto es, nueve personas casadas por cada persona en unión libre, lo cual es un porcentaje equivalente al muestral $(\chi 2[1, N=807]=0.97, p=.324)$.

\section{Instrumentos}

Se utilizó la MAS de O'Rourke y Cappeliez (2002), con la traducción de Moral (2007), según el método de traducción inversa. Consta de 18 ítems tipo Likert con un rango de respuestas de 1 (totalmente en desacuerdo) a 7 (totalmente de acuerdo). Catorce de sus 18 ítems son directos al rasgo del engrandecimiento, y cuatro son inversos (ítems 2, 11, 13 y 17 [Véase en el Anexo]). O'Rourke y Cappeliez (2002) reportaron una consistencia interna alta, que varió de .84 a $.87 \mathrm{al}$ ser estimada por el coeficiente alfa de Cronbach, y una estabilidad muy alta en un intervalo de quince meses $(r[200]=.80, p<.001)$. Hay dos modos de puntuar la escala. Se asigna 1 a los valores 6 y 7, salvo en los cuatro ítems inversos que esta asignación hace a los valores 1 y 2 ; y a los restantes valores se les asigna 0 . Al sumar los unos se tiene la puntuación en la escala dentro de un rango de 0 a 18 (O'Rourke \& Cappeliez, 2002). Otra forma es sumar el valor de respuesta dado a cada ítem, invirtiendo este valor en los ítems 2, 11, 13 y 17 (8-valor de respuesta), con lo que se logra la puntuación en la escala con un rango potencial de 1 a 126. En este caso la distribución de la escala se ajusta a una curva normal (Moral, 2007).

La RAS, con la validación en México por Moral (2015), está integrada por siete ítems tipo Likert, que tienen un rango de valores de uno a cinco. La puntuación se obtiene por suma simple de los ítems. Dos ítems están redactados en sentido contrario a satisfacción (ítems $4 \mathrm{y}$ 7). La escala presenta un recorrido potencial de 7 a 35. Una mayor puntuación refleja una mayor satisfacción con la relación de pareja. Moral (2015) obtuvo una consistencia interna excelente para los siete ítems de la RAS ( $\alpha$ ordinal $=.93$ ). El número de factores fue uno, obtenido por el análisis paralelo de Horn y la prueba de la correlación parcial media mínima de Velicer. El ajuste del modelo de un factor con 
todos sus residuos independientes fue bueno según mínimos cuadrados no ponderados $(\chi 2 / g l$ $=1.92, A G F I=.99, N F I=.99$ y $S R M R=.04)$.

\section{Procedimiento}

El estudio fue aprobado en sus aspectos éticos por el Comité de Doctorado, autoridad al respecto dentro de la Facultad de Psicología de la UANL), y financiado por el Programa de Apoyo a la Investigación Científica y Tecnológica de la UANL. Se solicitó el consentimiento expreso informado de los participantes en la primera hoja del cuestionario. En la hoja de consentimiento se garantizaba el anonimato de las respuestas y se informaba sobre la identidad del responsable del estudio, a quien se podía contactar por correo electrónico para cualquier cuestión suscitada por el estudio. No se solicitó ningún dato de identificación personal. De este modo se respetaron las normas éticas de investigación de la Asociación Americana de Psicología (APA, 2016).

\section{Análisis de datos}

En relación con el primer objetivo, a saber, estudiar las propiedades de los ítems, la discriminabilidad se determinó por la diferencia significativa de tendencia central en el ítem entre el grupo de puntuaciones altas y bajas en la escala. Las puntuaciones en la escala se crearon por la suma simple de los ítems (al invertir las puntuaciones de los dos ítems redactados en sentido opuesto al rasgo). Los grupos se definieron por el primer cuartil y el tercero en la escala. La diferencia de tendencia central se contrastó por la prueba $U$ de MannWhitney. La consistencia interna se estimó por la correlación del ítem con el resto de la escala, por el efecto de la eliminación del ítem sobre la consistencia interna de la escala y la comunalidad tras la extracción de un factor por el método de residuos mínimos o también denominado mínimos cuadrados no ponderados (ULS). La correlación se calculó con base en el coeficiente de correlación poliserial ( $r_{P S \text { [item, test- }}$ item] y la consistencia interna por el coeficiente alfa ordinal ( $\alpha$ test-ítem ordinal). Si $r_{P S \text { [item, test-item] }}$ $<.50$, se incrementa la consistencia interna de la escala con la eliminación del ítem ( $\alpha$ ordinal ${ }_{\text {test-item }}>\alpha$ ordinal $_{\text {test }}$ ) y la comunalidad del ítem es, entonces, menor que .25 , en ese caso se consideró que el ítem presentaba consistencia débil.

En relación con el segundo objetivo: calcular la consistencia interna de la escala, se usó el coeficiente alfa ordinal ( $\alpha$ ordinal) y el coeficiente alfa de Cronbach. Los valores de $\alpha$ de Cronbach y $\alpha$ ordinal $<.50$ evidencian una consistencia interna inaceptable, de .50 a .59 baja, de .60 a .69 cuestionable, de .70 a .79 aceptable, de .80 a .89 alta $y \geq .90$ excelente (Gadermann et al., 2014).

En relación con el tercer objetivo: determinar el número de factores, desde la matriz de correlaciones policóricas (estimadas por el método de dos pasos para máxima verosimilitud), se realizó el análisis paralelo de Horn (percentil 95 como criterio de intersección), se hizo el 
cálculo de promedios parciales al cuadrado de Velicer (promedio mínimo) y el análisis de coordenadas óptimas. El análisis de datos de comparación se hizo desde la matriz de correlaciones de Spearman, al no aceptar correlaciones policóricas (Courtney, 2013).

En relación con el cuarto objetivo: explorar la estructura factorial, se extrajeron los factores desde la matriz de correlaciones policóricas, según el método de residuos mínimos o, también denominado, mínimos cuadrados no ponderados (ULS). La rotación se realizó por el método Promax, se generó la hipótesis estructural por el método Varimax.

En relación con el quinto objetivo: contrastar los modelos factoriales propuestos, se aplicó Análisis Factorial Confirmatorio (AFC). Desde la matriz de correlaciones policóricas, la función de discrepancia se estimó por mínimos cuadrados libres de escala (SLS). El ajuste se valoró usando la chi-cuadrada relativa $(\chi 2 /$ $g l$ ), el índice de bondad de ajuste sin ajustar (Goodness of Fit Index, GFI, por sus siglas en inglés) y ajustado (Adjuested Goodness of Fit Index, AGFI, por sus siglas en inglés), el índice normado de ajuste (Normed Fit Index, NFI, por sus siglas en inglés), el índice relativo de ajuste o coeficiente $\rho 1$ de Bollen (Relative Fit Index, RFI, por sus siglas en inglés) y el residuo estandarizado cuadrático medio (Standardized Root Mean Squared Residual, SRMR, por sus siglas en inglés). Siguiendo a Byrne (2016), $\chi 2 / g l \leq 2, G F I, R F I$ y $N F I \geq .95, A G F I \geq .90$ y $S R M R \leq .05$ reflejan un ajuste bueno; y $\chi 2 / g l \leq$
3, GFI, RFI y $N F I \geq .90, A G F I \geq .85$ y $S R M R \leq$ .10 reflejan un ajuste adecuado. La significación de parámetros se estimó por el método de Percentiles Corregidos de Sesgo (PCS).

En relación con el sexto objetivo de comprobar la validez convergente, las correlaciones se estimaron por el coeficiente de correlación producto-momento de Pearson $(r)$. Valores de $r<.30$ se consideraron correlaciones bajas, de .30 a .49 moderadas, de .50 a .69 altas, de .70 a .89 muy altas $\mathrm{y} \geq .90$ unitarias (Bosco, Aguinis, Singh, Field, \& Pierce, 2015). Los cálculos se realizaron con $S P S S$, versión 22, menú $\mathrm{R}$, versión 2.4 para SPSS 22, y con AMOS, versión 16.

\section{Resultados}

\section{Distribución, discriminación y consistencia de los 18 ítems de la MAS}

Ninguno de los ítems presentó efecto suelo (concentración de $90 \%$ o más de su distribución en el valor inferior) ni efecto techo (concentración de $90 \%$ o más de su distribución en el valor superior). Respecto a los ítems directos, las medianas de los ítems 1, 3, 4, 5, 9, 14 y 18 quedaron en el polo de la aceptación del engrandecimiento (valores 5, "bastante de acuerdo", o 6, "totalmente de acuerdo"), las de los ítems 6, 7 y 8 (tres de los 14 ítems) en el polo del rechazo del engrandecimiento (3, "más bien en desacuerdo", o 2, "bastante en desacuerdo") y las de los ítems 10, 12, 15 y 16 (cuatro de los 14 ítems) en un valor ambiguo ("ni de acuerdo ni en desacuerdo"). De los 
cuatro ítems inversos, la mediana del ítem 2 se inclinó hacia el polo del desacuerdo $(M d n=3)$; las medianas de los ítems 11 y 17 se inclinaron hacia el polo de la aceptación de la afirmación contraria al engrandecimiento $(M d n=5)$. El ítem 13 tuvo una media de 4 ("ni de acuerdo ni en desacuerdo"), pero su asimetría negativa $(\mathrm{AI}=-.33)$ pone a su mediana más próxima al tercer cuartil (5) que al primero (2), esto es, lo posiciona hacia el rechazo del engrandecimiento (tabla 2).

Tabla 2

Distribución, discriminación, consistencia interna y validez de los ítems

\begin{tabular}{|c|c|c|c|c|c|c|c|c|c|}
\hline \multirow{2}{*}{ Ítems } & \multicolumn{4}{|c|}{ Descriptivos } & \multirow{2}{*}{$\frac{\text { Discrim. }}{Z}$} & \multicolumn{3}{|c|}{ Consistencia interna } & \multirow{2}{*}{$\begin{array}{l}\text { Validez } \\
r_{P S(\mathrm{i}, \mathrm{RAS})}\end{array}$} \\
\hline & $M d n$ & $R S I$ & $A_{1}$ & $C_{p c}$ & & $r_{P S(\mathrm{i}, \mathrm{t}-\mathrm{i})}$ & $\alpha_{(t-i)}$ & Com. & \\
\hline MAS1 & 6 & 1.5 & -.33 & 0.04 & $-15.58^{* * *}$ & $.61^{* * *}$ & .83 & .51 & $.54^{* \star \star}$ \\
\hline MAS2* & 3 & 2 & 0 & 0.14 & $-11.08^{* * *}$ & $-.37^{\star * *}$ & .84 & .18 & $-.49^{* * *}$ \\
\hline MAS3 & 5 & 1.5 & .33 & 0.04 & $-15.55^{\star \star \star}$ & $.61^{* * *}$ & .83 & .51 & $.51^{\star \star \star}$ \\
\hline MAS4 & 6 & 1.5 & -.33 & 0.04 & $-13.56^{\star \star *}$ & $.50^{* * *}$ & .84 & .33 & $.40^{* * *}$ \\
\hline MAS5 & 5 & 1 & 0 & -0.02 & $-9.13^{\star \star *}$ & $.34^{*+*}$ & .84 & .17 & $.22^{* \star *}$ \\
\hline MAS6 & 3 & 1.5 & -.33 & 0.11 & $-13.22^{* * *}$ & $.48^{* * *}$ & .84 & .21 & $.30^{* * *}$ \\
\hline MAS7 & 3 & 2 & 0 & 0.14 & $-7.58^{* * * x}$ & $.26^{*+*}$ & .85 & .06 & $.07^{\mathrm{ns}}$ \\
\hline MAS8 & 2 & 1.5 & .33 & 0.11 & $-11.70^{* \star *}$ & $.41^{* * *}$ & .84 & .12 & $.17^{\star \star \star}$ \\
\hline MAS9 & 5 & 1 & 0 & -0.06 & $-16.29^{\star \star \star}$ & $.70^{* * *}$ & .83 & .60 & $.60^{\star \star *}$ \\
\hline MAS10 & 4 & 1.5 & .33 & -0.01 & $-13.69^{* * *}$ & $.53^{*+*}$ & .83 & .32 & $.39^{* * *}$ \\
\hline MAS11* & 5 & 1 & 0 & -0.06 & $-4.85^{\star \star \star *}$ & $-.11^{* *}$ & .85 & .01 & $-.06^{\mathrm{ns}}$ \\
\hline MAS12 & 4 & 2 & 0 & 0.07 & $-12.08^{\star \star \star}$ & $.43^{* * *}$ & .84 & .25 & $.29^{* \star *}$ \\
\hline MAS13* & 4 & 1.5 & -.33 & 0.04 & $-7.09^{* * * *}$ & $-.23^{* * *}$ & .85 & .06 & $-.26^{\star \star *}$ \\
\hline MAS14 & 5 & 2 & 0 & 0.07 & $-15.47^{\star \star \star}$ & $.66^{\star \star \star *}$ & .83 & .57 & $.51^{\star \star \star *}$ \\
\hline MAS15 & 4 & 1.5 & .33 & -0.01 & $-9.69^{\star \star *}$ & $.37^{* * *}$ & .84 & .20 & $.22^{\star \star \star}$ \\
\hline MAS16 & 4 & 1.5 & .33 & -0.01 & $-11.68^{* \star *}$ & $.44^{* * *}$ & .84 & .23 & $.25^{\star \star *}$ \\
\hline MAS17* & 5 & 1.5 & .33 & -0.01 & $-4.89^{* * * x}$ & $-.13^{* * *}$ & .85 & .01 & $-.17^{* \star *}$ \\
\hline MAS18 & 6 & 1 & 0 & -0.01 & $-15.14^{* * *}$ & $.67^{* \star *}$ & .83 & .62 & $.68^{* * *}$ \\
\hline
\end{tabular}

Notas. $N=807$. ${ }^{*}$ Ítems inversos. ${ }^{* *} p \leq .001,{ }^{* *} p \leq .01{ }^{*} p \leq .05, \mathrm{~ns}=p>.05 . \mathrm{a}_{(\mathrm{t}-\mathrm{i})}=$ coeficiente alfa ordinal calculado sin incluir al ítem. $\alpha$ ordinal $_{(\mathrm{t})}=.846$ (para su cálculo se invirtieron las puntuaciones de los ítems $2,11,13$ y 17 y se incluyeron los 18 ítems). Fuente: elaboración propia.

Los 18 ítems fueron discriminativos $(p<$ $.001)$, de donde los ítems inversos 11 y 17 tuvieron la menor diferencia $\left(Z_{U}>-5\right)$ entre los dos grupos de puntuaciones extremas, definidos por el primer cuartil $\left(Q_{1}=65\right)$ y el tercero $\left(Q_{3}=\right.$ 88) (tabla 2). 
Los 18 ítems se correlacionaron con el resto de la escala, pero los ítems 7, 11, 13 y 17 tuvieron correlaciones bajas. La consistencia interna de los 18 ítems fue alta ( $\alpha$ ordinal $_{\text {[test] }}=.85$ ); ésta se incrementó con la eliminación de los ítems 11, 17 y 13 ( $\alpha$ ordinal $_{\text {[test] }}=.87$ ) y no se alteró con la eliminación del ítem 7. A su vez, los ítems 7, 11,13 y 17 tuvieron comunalidades $<.10$. Al estimar la consistencia por el coeficiente alfa de Cronbach, también resultó alta ( $\alpha$ de Cronbach $=.816)$ y se incrementó con la eliminación de los ítems $11(\alpha$ de Cronbach $=.817), 13(\alpha$ de Cronbach $=.825)$ y $17(\alpha$ de Cronbach $=.823)$ (tabla 2).

Todos los ítems se correlacionaron con la RAS, salvo los ítems 7 y 11. Los ítems 1, 3, 9, 14 y 18 tuvieron correlaciones altas (tabla 2).

Del análisis de los ítems, se evidenciaron claros problemas de consistencia y validez en los ítems inversos 11, 13 y 17 y en el ítem directo 7, que quedaron en el polo de rechazo de las afirmaciones de engrandecimiento y fueron de los menos discriminativos.

\section{Número de factores $y$ exploración de la estructura factorial}

Desde la matriz de correlaciones policóricas de los 18 ítems, el número de factores fue dos por el criterio de Velicer y tres por los criterios de Horn, coordenadas óptimas y el análisis de datos de comparación.

Al extraer un factor conforme a la expectativa (O'Rourke, \& Cappeliez, 2002), se explicó $27.6 \%$ de la varianza. Los ítems 7, 11, 13 y 17 presentaron saturaciones $<.30$. La consistencia interna de los 14 ítems que configuraron el factor fue alta ( $\alpha$ ordinal $=.87$ y $\alpha$ de Cronbach $=.84$ con el ítem 2 invertido).

Al extraer dos factores conforme al criterio de Velicer y la expectativa (Moral, 2007), se explicó $35.3 \%$ de la varianza total. Tras la rotación, los ítems 11, 13 y 17 presentaron saturaciones $<.30$. Se definió un primer factor configurado por doce indicadores de sentimientos positivos (ítems 1,2, $3,4,5,9,10,12,14,15,16$ y 18) con consistencia interna alta $(\alpha$ ordinal $=.87$ y $\alpha$ de Cronbach $=$ .84 con el ítem 2 invertido), y un segundo factor con tres indicadores de sentimientos y eventos negativos pocos frecuentes (ítems 6, 7 y 8) con consistencia interna aceptable por el coeficiente alfa ordinal ( $\alpha$ ordinal $=.74)$ y cuestionable por el coeficiente alfa de Cronbach $(\alpha=.68)$. La correlación entre ambos factores fue moderada y positiva $(r=.45, \mathrm{p}<.001)$.

$\mathrm{Al}$ extraer tres factores conforme a la convergencia de los criterios de Horn, datos de comparación y coordenadas óptimas, se explicó $40.1 \%$. Los 18 ítems tuvieron cargas $\geq .30$ o $\leq-.30$. Tras la rotación, se definió un primer factor con once indicadores sobre cualidades y sentimientos positivos evaluados con ítems directos (ítems 1, 3, 4, 5, 9, 10, 12, 14, 15, 16 y 18) con consistencia interna alta ( $\alpha$ ordinal $=.87$ y $\alpha$ de Cronbach $=.84$ ); un segundo factor con cuatro indicadores sobre sentimientos y eventos negativos (ítems 6, 7, 8 y 11) con consistencia interna cuestionable por el coeficiente alfa ordinal $(\alpha$ ordinal $=.68$ con el ítem 11 invertido), 
que mejoró a aceptable al eliminar el ítem $11(\alpha$ ordinal $=.74)$, y baja por el coeficiente alfa de Cronbach $(\alpha=.59)$, que pasó a cuestionable con la eliminación del ítem $11(\alpha=.68)$; y un tercer factor con tres indicadores sobre cualidades positivas evaluadas con ítems inversos (ítems 2,13 y 17) con consistencia interna baja por el coeficiente alfa ordinal ( $\alpha$ ordinal $=.51$ ) $\mathrm{e}$ inaceptable por el coeficiente alfa de Cronbach $(\alpha=.48)$. La correlación entre los dos primeros factores fue moderada y positiva $(r=.42, p<$ .001) y las correlaciones con el tercer factor fueron significativas, pero bajas.

Al eliminar los cuatro ítems con problemas de consistencia interna y validez convergente (ítems 7, 11, 13 y 17), el número de factores fue uno por el criterio de Velicer y dos por los criterios de Horn, coordenadas óptimas y análisis de datos de comparación.

Al extraer un factor se explicó $34.4 \%$ de la varianza total y todos los ítems tuvieron cargas $>.300<-.30$. La consistencia interna entre los catorce ítems fue alta ( $\alpha$ ordinal $=.87$ y $\alpha$ de Cronbach $=.84)$. Al extraer dos factores, se explicó $41.6 \%$ de la varianza total. Los catorce ítems tuvieron cargas $\geq .30 \mathrm{o} \leq-.30$. Tras la rotación, el primer factor quedó configurado por doce indicadores sobre sentimientos positivos de enamoramiento, comprensión y satisfacción (ítems 1, 2, 3, 4, 5, 9, 10, 12, 14, 15, 16 y 18) con consistencia interna alta ( $\alpha$ ordinal $=.87 \mathrm{y}$ $\alpha$ de Cronbach $=.84$ con el ítem 2 invertido).
El segundo factor quedó configurado por dos indicadores sobre sentimientos $y$ eventos negativos pocos frecuentes (ítems 6 y 8) con consistencia interna aceptable por la alfa ordinal $(\alpha$ ordinal $=.72)$ y cuestionable por la alfa de Cronbach $(\alpha=.64)$. La correlación entre ambos factores fue alta y positiva $(r=.55, p<.001)$.

Del análisis de los ítems y resultado del análisis factorial exploratorio, se deberían eliminar los ítems 7, 11, 13 y 17 con problemas de consistencia interna y validez para definir un factor único, pero se podría conservar el ítem directo 7 para definir un modelo de dos factores. El modelo de tres factores puede ser desestimado por la consistencia interna baja del tercer factor conformado por tres ítems con problemas de consistencia interna y validez.

\section{Análisis factorial confirmatorio}

Se contrastaron tres modelos con todos sus residuos de medida independientes (tabla 3 ). Por una parte, se especificó el modelo de un factor con los 18 indicadores originales (F1_18) y con los 14 indicadores seleccionados desde el análisis de los ítems y el análisis factorial exploratorio (F1_14). Por otra parte, se especificó el modelo de dos factores correlacionados con 15 ítems seleccionados desde el análisis de los ítems y el análisis factorial exploratorio (F2_15): el factor de sentimientos/eventos positivos con 11 indicadores y el factor de sentimientos/eventos negativos con tres indicadores, que es afín al modelo bifactorial hipotetizado (Moral, 2007). 
Tabla 3

Índices de ajuste

\begin{tabular}{lccccc}
\hline \multirow{2}{*}{ Índices } & \multicolumn{2}{c}{ Interpretación } & \multicolumn{3}{c}{ Modelos } \\
\cline { 2 - 5 } & Bueno & Malo & F1_18 & F1_14 & F2_15 \\
\hline$X^{2}$ & & & 974.878 & 361.262 & 303.764 \\
$g l$ & & 135 & 77 & 89 \\
$X^{2} / g l$ & $\leq 2$ & 7.221 & 4.692 & 3.413 \\
$G F I$ & $\geq .95$ & $\geq .90$ & .944 & .975 & .980 \\
$A G F I$ & $\geq .90$ & $\geq .85$ & .929 & .966 & .974 \\
$N F I$ & $\geq .95$ & $\geq .90$ & .903 & .959 & .968 \\
$R F I$ & $\geq .95$ & $\geq .90$ & .891 & .952 & .962 \\
$S R M R$ & $\leq .05$ & $\leq .10$ & .084 & .065 & .056 \\
\hline
\end{tabular}

Método: mínimos cuadrados libres de escala. Modelos con todos los residuos de medida independientes: F1_18 = de un factor con 18 indicadores, F1_14 = de un factor con catorce indicadores y F2_15 = de dos factores correlacionados con tres indicadores un factor y doce el otro factor. Fuente: elaboración propia.

La solución del modelo de un factor con 18 indicadores (F1_18) fue admisible. Todos los parámetros fueron significativos, salvo el peso del factor general sobre el ítem 11. Los pesos estandarizados del factor sobre los 18 ítems variaron de -.41 (ítem 2) a .78 (ítem 9) con una media en valor absoluto de .48 (figura 1). De los seis índices de ajuste contemplados, uno fue bueno $(A G F I=.93)$, tres fueron aceptables $(G F I$ $=.94, N F I=.90$ y $S R M R=.08)$ y dos fueron malos $(\chi 2 / g l=7.22$ y $R F I=.89)($ tabla 3$)$.

La solución del modelo de un factor con catorce indicadores (F1_14) fue admisible. Todos los parámetros fueron significativos. Los pesos estandarizados en valores absolutos del factor general sobre los catorce ítems variaron de .36 (ítem 8) a .78 (ítem 9) con una media de.57 (figura 2). De los seis índices de ajuste contemplados, cuatro fueron buenos $(G F I=.98, A G F I$
$=.97, N F I=.96 \mathrm{y} R F I=.95) \mathrm{y}$ dos fueron aceptables $(\chi 2 / g l=4.69$ y $S R M R=.07)$. La bondad del modelo de un factor con catorce indicadores de ajuste fue significativamente mejor que la del modelo de un factor con 18 indicadores $(\Delta \chi 2[58]$ $=613.62, p<.001)($ tabla 3$)$.

La solución del modelo de dos factores correlacionados (F2_15) fue admisible. Todos los parámetros fueron significativos. La media de los pesos estandarizados en valores absolutos del factor de sentimientos/eventos positivos sobre sus once indicadores fue .60 y los pesos variaron de .40 (ítem 2) a .78 (ítem 9). La media de los pesos estandarizados del factor de sentimientos/eventos negativos sobre sus tres indicadores fue $.70 \mathrm{y}$ los pesos variaron de .52 (ítem 7) a .86 (ítem 6). La correlación entre los dos factores fue moderada $(r=.46$; IC $95 \%$ : .39, $.52 ; p=.001$ ) (figura 3). De los seis índices de 
ajuste contemplados, cuatro fueron buenos ( $G F I$ $=.98, A G F I=.97, N F I=.97$ y $R F I=.96)$ y dos fueron aceptables $(\chi 2 / g l=3.41$ y $S R M R=.06)$. La bondad de ajuste del modelo de dos factores correlacionados fue significativamente mejor que la del modelo de un factor con catorce indicadores $(\Delta \chi 2[12]=57.50, p<.001)($ tabla 3$)$.

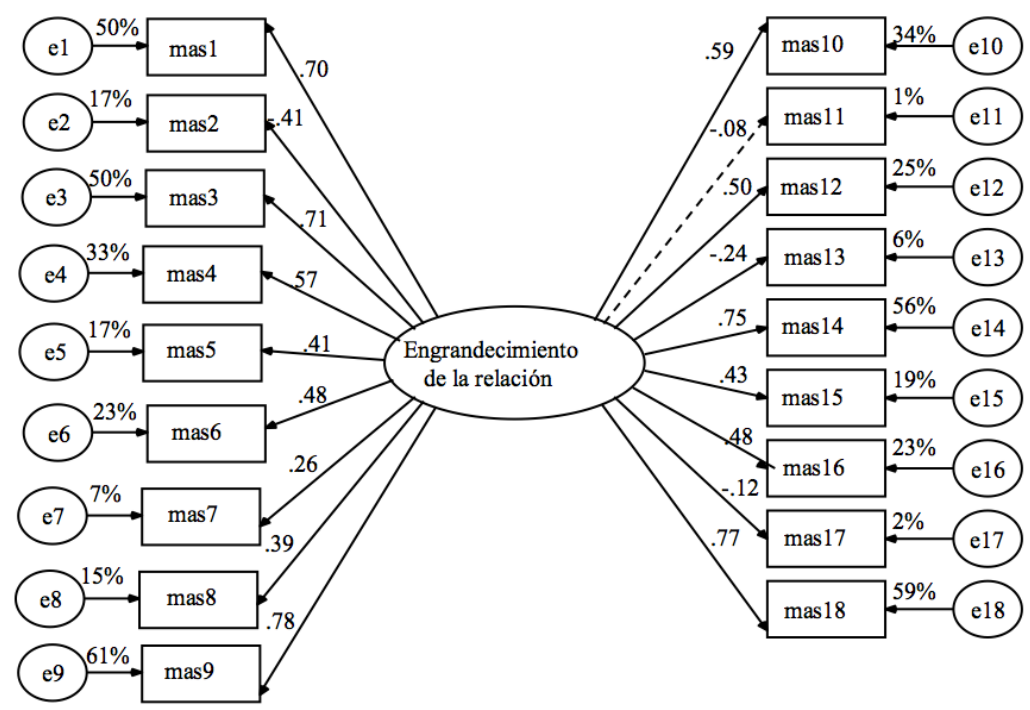

Figura 1. Modelo de un factor con 18 indicadores. Fuente: elaboración propia.

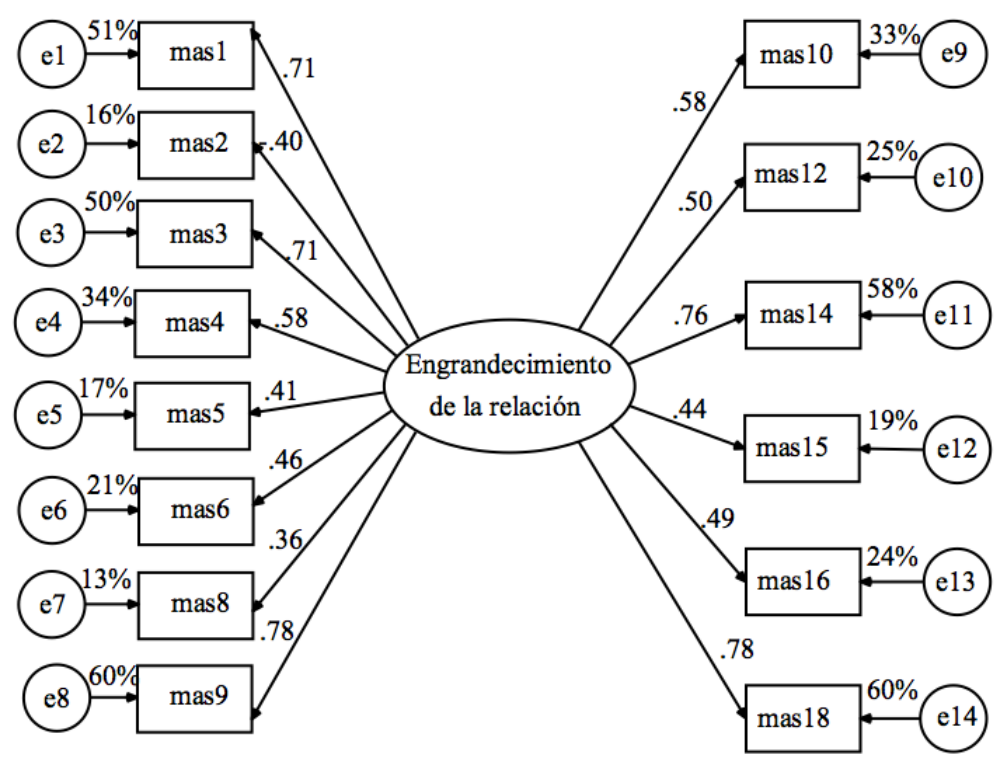

Figura 2. Modelo de un factor con catorce indicadores. Fuente: elaboración propia. 


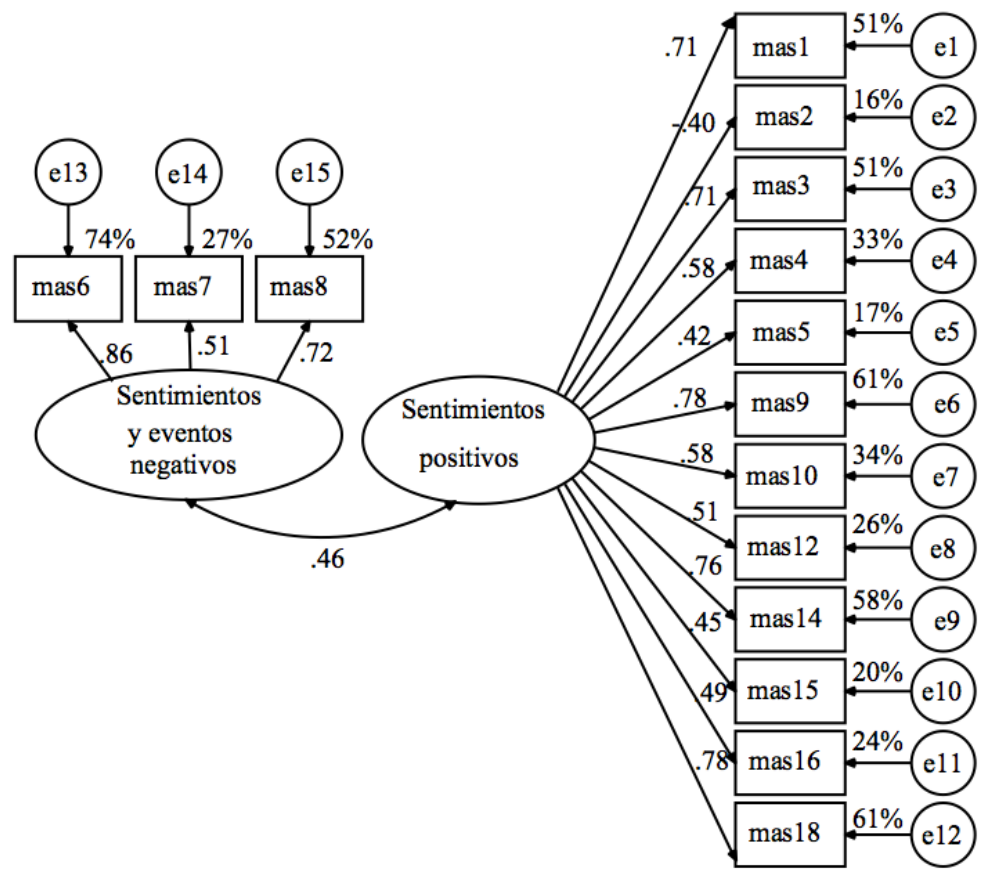

Figura 3. Modelo de dos factores correlacionados con quince ítems.

Fuente: elaboración propia.

\section{Validez convergente con satisfacción con la} relación de pareja

En la muestra conjunta, las correlaciones de la RAS fueron significativas, positivas y altas con las puntuaciones totales en la escala de engrandecimiento (MAS, MAS_15 y MAS_14) $\mathrm{y}$ con el factor de sentimientos y eventos positivos, variando de .56 a .66 , y fue baja con el factor de sentimientos y eventos negativos $(r$ $=.26, p<.001)$.

\section{Discusión}

En relación con el primer objetivo de estudiar las propiedades psicométricas de los 18 ítems de la MAS, se observaron problemas de consistencia interna y correlación no significativa o baja con satisfacción con la relación de pareja en los ítems inversos 13 y 17, como se esperaba (Moral, 2007), así como en el ítem inverso $11 \mathrm{y}$ en el ítem directo 7 ("si mi pareja tiene defectos no soy consciente de ellos"). De los cuatro ítems inversos, sólo el ítem 2 presentó unas propiedades buenas de distribución (simétrica), discriminación, consistencia y validez convergente. Su comunalidad inicial fue algo baja $(<.20)$, pero en el modelo de un factor y en el de dos factores presentó una comunalidad final mayor que .20 , por ende, se podría mantener. Respecto a los otros tres 
ítems inversos y el ítem directo 7, sería mejor eliminarlos para definir un modelo de un factor. Los ítems inversos parecen generar confusión, sobre todo al considerar que el constructo que se evalúa es engrandecimiento. La negación de unas pocas cualidades positivas, en un contexto de afirmación de cualidades positivas y negación de defectos parece ocasionar confusión y dificultades de entendimiento, lo cual pudiera explicar los problemas de consistencia interna y validez.

Considerando el modelo de un factor con catorce indicadores, el promedio de la mayoría de los ítems retenidos quedó en el polo del engrandecimiento (ocho de los catorce ítems), incluyendo el ítem inverso 2, el promedio de cuatro ítems correspondió al intervalo de respuesta ambigua (ni acuerdo ni en desacuerdo) y el promedio de dos ítems quedó en el polo del rechazo del engrandecimiento. La polaridad de los ítems retenidos se inclinó hacia el engrandecimiento sin ser ésta una polaridad extrema y sin que ninguno de ellos se acerque al efecto techo. Precisamente, la distribución de la mayoría de los ítems fue simétrica (seis de los catorce ítems) o asimétrica positiva (cinco de los catorce ítems) y mesocúrtica $(>-.1 \mathrm{y}<.1)$. Debe señalarse que los ítems que presentaron rechazo del engrandecimiento fueron los que definieron el segundo factor en el modelo bifactorial, y se refieren a la ausencia de sentimientos y eventos negativos.

En relación con el segundo objetivo de estimar la consistencia interna de la MAS, se confirmó la expectativa de consistencia interna alta entre los 18 ítems o los catorce retenidos para definir el modelo de un factor, tanto si el cálculo se realiza por el coeficiente alfa de Cronbach como por el coeficiente alfa ordinal, siendo la estimación más alta por el coeficiente alfa ordinal, que es un coeficiente más adecuado para datos ordinales (Elosua, \& Zumbo, 2008).

La estimación en la presente muestra poblacional por el coeficiente alfa de Cronbach fue más alta que la obtenida dentro de la misma población por la muestra incidental por Moral (2007), siendo la diferencia de cinco décimas. Por las características del muestreo, debe concederse mayor valor a la presente estimación que a la anterior, realizada en una muestra incidental más pequeña. No obstante, ambas estimaciones en México fueron más bajas que las obtenidas por O'Rourke et al. (2012) en muestras canadienses anglófonas de adultos mayores, que la obtenida por O'Rourke et al. (2012) en una muestra israelita de adultos mayores, y que la obtenida además, por O'Rourke y Cappeliez (2003) en una muestra canadiense francófona de adultos mayores.

Estos datos parecen reflejar que la consistencia de la MAS en las poblaciones de adultos mayores canadienses e israelitas es más alta que en población general de adultos mexicanos. En la presente muestra, la media en la MAS (en un rango de 0 a 18) de los doce participantes de 60 años o más $(M=7.50$, IC $95 \%: 5.45,9.55)$ fue estadísticamente equivalente a la media de los 795 participantes menores de 60 años $(M=5.87$, 
IC 95\%: 5.59, 6.15) por la prueba $t$ de Student con la corrección de Welch $(t[11.52]=1.73, p$ $=.111)$, y el valor de la consistencia interna fue semejante entre los doce adultos mayores $(\alpha$ de Cronbach $=.816$ y $\alpha$ ordinal $=.850)$ y el resto de los 715 adultos ( $\alpha$ de Cronbach $=.818$ y $\alpha$ ordinal $=.847$ ), por consiguiente, la edad no parece contribuir a la diferencia de consistencia.

La menor consistencia interna pudiera atribuirse a dificultades de entendimiento de los ítems inversos en las muestras mexicanas, ya que tanto en las muestras canadienses como en la muestra israelita los ítems inversos no presentaron problemas de consistencia interna, salvo el ítem 17 (O'Rourke, \& Cappeliez, 2002; O'Rourke et al., 2012). Debe señalarse que el promedio de escolaridad de la muestra mexicana fue menor que el de las muestras de adultos mayores canadienses e israelitas con una diferencia de tres años. Aunque la MAS fue independiente de los años de escolaridad en las muestras canadienses e israelitas, esta correlación fue significativa en la presente muestra y la consistencia entre los 18 ítems fue menor entre los 404 participantes con estudios de secundaria o menores ( $\alpha$ de Cronbach $=.77 \mathrm{y}$ $\alpha$ ordinal $=.80)$ que entre los 403 participantes con estudios de preparatoria o mayores ( $\alpha$ de Cronbach $=.83$ y $\alpha$ ordinal $=.86)$, por ende, la menor escolaridad afecta la consistencia de las respuestas, seguramente por la mayor dificultad para comprender los ítems inversos.

En relación con el tercer objetivo de determinar el número de factores por la convergencia de los criterios, no se sostuvo la hipótesis de un modelo de un factor con los 18 ítems (O'Rourke, \& Cappeliez, 2002; O'Rourke et al., 2012). Esta hipótesis sólo se podría fundamentar por el criterio de Velicer, tras eliminar los cuatro ítems con problemas de consistencia interna. No obstante, después de dicha eliminación, la convergencia se produjo en dos factores, conforme con la expectativa derivada del estudio de Moral (2007).

En relación con el cuarto objetivo de explorar la estructura factorial en caso de que el número de factores fuese mayor que uno, y estimar la consistencia interna de estos factores, se reproduce la configuración de los dos factores reportada por Moral (2007), aunque con mejores resultados de consistencia interna. Estimada la consistencia interna por el coeficiente alfa de Cronbach, el factor de sentimientos positivos tuvo una consistencia interna alta en la presente muestra, cuando fue aceptable en el anterior estudio. El factor de sentimientos y eventos negativos pocos frecuentes tuvo una consistencia interna cuestionable en ambos estudios, pero el valor fue siete décimas más alto en la presente muestra, siendo aceptable al ser estimado por el coeficiente alfa ordinal. Como ya se indicó, el hecho de que la presente muestra sea de rutas al azar da más confiabilidad a la estimación que la del estudio de Moral (2007), con una muestra no probabilística y de menor tamaño.

En relación con el quinto objetivo de contrastar el ajuste del modelo de un factor con los residuos de medida independientes, se logró 
un ajuste aceptable una vez que se eliminaron los cuatro ítems con problemas de consistencia interna y validez convergente. También, al eliminar tres de los cuatro ítems con problemas de consistencia interna y validez convergente, se obtuvo un ajuste aceptable del modelo de dos factores, especificado de forma equivalente al de Moral (2007). El modelo bifactorial mostró mayor bondad de ajuste que el de un factor con catorce indicadores. Los dos factores fueron discernibles, al compartir menos de un cuarto de la varianza, además, poseen un significado teórico.

Se podría entender desde el modelo bifactorial de las emociones que postula, la existencia de dos predisposiciones afectivas diferenciables, al afecto negativo (miedo, tristeza, ira, culpa) y predisposición al afecto positivo (alegría, entusiasmo, contento) que permiten entender diversos trastornos afectivos y motivacionales (Burgess, \& Nakamura, 2014). La cualidad positiva o negativa de la emoción parece afectar la expresión del engrandecimiento, siendo su expresión más consistente y más definida ante el afecto positivo, y dicho efecto no es atribuible a un artefacto de ítems directos e inversos (Molina, Rodrigo, Losilla, \& Vives, 2014), ya que el sentido de la redacción del ítem (directo o inverso) no contribuye a separar a un factor del otro en el formato original de 18 ítems; además, tres de los cuatro ítems inversos fueron eliminados en el formato final. La debilidad del modelo bifactorial es la consistencia del factor de emociones/eventos negativos, la cual resultó aceptable por el coeficiente alfa ordinal y cuestionable por el coeficiente alfa de Cronbach, no alcanzó un nivel bueno. Tal vez al incrementar su número de indicadores se podría aumentar su consistencia interna (Gadermann et al., 2014). Cabría señalar que uno de sus indicadores, el ítem 7 , fue consistente dentro del factor, pero no dentro de la escala, asimismo, fue independiente de la satisfacción con la relación de pareja, por lo cual sería conveniente sustituirlo.

En relación con el sexto objetivo de estudiar la validez convergente de la MAS, se confirmó la expectativa de correlación alta con satisfacción con la relación de pareja, de donde la varianza compartida resultó menor que la mitad. Un resultado afín, con respecto al ajuste diádico, ha sido reportado en muestras canadienses anglófonas (O'Rourke, \& Cappeliez, 2002), en una muestra canadiense francófona (O’Rourke, $\&$ Cappeliez, 2003) y en una muestra mexicana (Moral, 2007), lo cual indica que son dos conceptos relacionados, pero distinguibles.

Como limitación del estudio debe señalarse que su generalización sólo es válida para la población de parejas casadas o en unión libre de la ciudad de Monterrey en México. En otras poblaciones urbanas de México o de países económica y culturalmente afines deben manejarse como hipótesis. Muestras extraídas de población rural, clínica o de novios pueden diferir más en sus características, en tal caso, los presentes datos servirían de comparación.

En conclusión, tres de los cuatro ítems inversos $\mathrm{y}$ el ítem directo 7 presentaron 
problemas de consistencia interna y validez. Con la eliminación de los ítems anteriores, el modelo esperado de un factor se justificó por el criterio de Velicer y presentó un ajuste aceptable, manteniéndose los residuos de medida independientes. La consistencia interna de los catorce ítems retenidos fue alta. Sin necesidad de eliminar el ítem 7, la convergencia de criterios para determinar el número de factores se produjo en dos. Se reprodujo, de igual forma, el modelo esperado de sentimientos positivos y sentimientos y eventos negativos poco frecuentes, con consistencia interna alta el primero y aceptable el segundo, al ser estimada por el coeficiente alfa ordinal; su ajuste a los datos fue aceptable y ligeramente mejor que el de un factor.

Se recomienda el uso del modelo de un factor, conforme con la teoría del engrandecimiento de O'Rourke y Cappeliez (2002), pero reduciéndolo a catorce indicadores. Incluso la redacción del ítem 2 se podría hacer directa ("mi matrimonio ha sido un éxito perfecto"), con lo que probablemente mejorare el ajuste del modelo unifactorial, la consistencia interna del ítem y la escala. Esta escala breve sería útil para estudiar y evaluar procesos de adaptación y superación de crisis en las parejas (Liu, Wang, \& Jackson, 2017).

El modelo de dos factores requeriría fortalecimiento de indicadores (de cuatro a seis indicadores) para alcanzar una consistencia interna alta, e incluso sustituir al ítem 7 poco consistente con la escala, e independiente de la satisfacción con la relación de pareja. Este modelo podría relacionarse teóricamente con el modelo bifactorial de las emociones, por lo tanto, se sugiere seguir desarrollando la MAS en sostén del modelo bifactorial y profundizar en el mismo.

\section{Referencias}

American Psychological Association. (2016). Ethical principles of psychologists and code of conduct (third revision). Washington, DC: APA.

Bosco, F. A., Aguinis, H., Singh, K., Field, J. G., \& Pierce, C. A. (2015). Correlational effect size benchmarks. Journal of Applied Psychology, 100, 431-449. doi:10.1037/ a0038047

Burgess, A. M., \& Nakamura, B. J. (2014). An Evaluation of the Two-Factor Model of Emotion: Clinical Moderators Within a Large, Multi-Ethnic Sample of Youth. Journal of Psychopathology and Behavioral Assessment, 36, 124-135. doi:10.1007/ s10862-013-9376-z

Byrne, B. M. (2016). Structural equation modeling with AMOS: Basic concepts, applications, and programming. (3a ed.). New York, NY: Routledge.

Courtney, M. G. R. (2013). Determining the number of factors to retain in EFA: Using the SPSS R-Menu v2.0 to make more judicious estimations. Practical Assessment Research and Evaluation, 18(8), 24-57. 
Elosua, P., \& Zumbo, B. (2008). Coeficientes de fiabilidad para escalas de respuesta categórica ordenada. Psicothema, 20(4), 896-901.

Gadermann, A., Guhn, M., \& Zumbo, B. D. (2014). Ordinal alpha. En A. C. Michalos (Ed.) Encyclopedia of quality of life and wellbeing research (pp. 4513-4515). Dordrecht, Netherlands: Springer. doi:10.1007/978-94007-0753-5

Gaskin, C. J., \& Happell, B. (2014). On exploratory factor analysis: A review of recent evidence, an assessment of current practice, and recommendations for future use. International Journal of Nursing Studies, 51, 511-521. doi: 10.1016/j.ijnurstu.2013.10.005 Hayduk, L. (2014). Seeing perfectly fitting factor models that are causally misspecified understanding that close-fitting models can be worse. Educational and Psychological Measurement, 74, 905-926. doi:10.1177/0013164414527449

Hendrick, S. S. (1988). A generic measure of relationship satisfaction. Journal of Marriage and the Family, 50, 93-98. doi: $10.2307 / 352430$

Instituto Nacional de Estadística, Geografía e Informática (2016). Panorama sociodemográfico de Nuevo León 2015. Ciudad de México: INEGI.

Liu, J., Wang, Y., \& Jackson, T. (2017). Towards explaining relationship dissatisfaction in Chinese dating couples: Relationship disillusionment, emergent distress, or insecure attachment style? Personality and Individual Differences, 112, 42-48. doi:10.1016/j.paid.2017.02.041

Molina, J. G., Rodrigo, M. F., Losilla, J. M., \& Vives, J. (2014). Wording effects and the factor structure of the 12-item General Health Questionnaire (GHQ-12). Psychological Assessment, 26, 1031-1037. doi:10.1037/ a0036472.

Moral, J. (2007). Control de la deseabilidad social ante la evaluación del ajuste diádico y la satisfacción marital. Psicología y Ciencia Social, 9(2), 5-12.

Moral, J. (2015). Validación del modelo unidimensional de la Escala de Valoración de la Relación en Personas Casadas y en Unión Libre de Monterrey, México. Revista Internacional de Psicología, 14(2), 1-70.

O'Rourke, N., \& Cappeliez, P. (2002). Development and validation of a couples measure of biased responding: The Marital Aggrandizement Scale. Journal of Personality Assessment, 78, 301-320. doi:10.1207/S15327752JPA7802_06

O'Rourke, N., \& Cappeliez, P. (2003). Validation d'une mesure de réponses biaisées à propos de la relation conjugale: L'Échelle d'embellissement conjugal. Revue Canadienne des Sciences du Comportement, 35, 45-49. doi:10.1037/h0087185

O'Rourke, N., Polchenko, N., Bachner, Y. G., \& Carmel, S. (Agosto, 2012). Translation and validation of a Hebrew language version of the Marital Aggrandizement Scale. Trabajo presentado en the Annual Meeting of The 
American Psychological Association, Orlando, FL. Recuperado de http://www. researchgate.net/publication/252931460

Pick, S., \& Andrade, P. (1988). Desarrollo y validación de la Escala de Satisfacción Marital (ESM). Psiquiatría, 4(1), 9-20.

Spanier, G. B. (1976). Measuring dyadic adjustment: New scales for assessing the quality of marriage and similar dyads. Journal of Marriage and the Family, 38, 1528. doi:10.2307/350547

Valdivieso, C. E. (2013). Efecto de los métodos de estimación en las modelaciones de estructuras de covarianzas sobre un modelo estructural de evaluación del servicio de clases. Comunicaciones en Estadística, 6(1), 21-44.

West, T. V., Dovidio, J. F., \& Pearson, A. R. (2014). Accuracy and bias in perceptions of relationship interest for intergroup and intragroup roommates. Social Psychological and Personality Science, 5, 235-242. doi:10.1177/1948550613490966

Recibido el 21 de febrero de 2017

Revisado el 28 de abril de 2017

Aceptado el 01 de mayo de 2017 


\section{Apéndice}

\section{Escala de Engrandecimiento Marital (MAS)}

Lea atentamente las siguientes afirmaciones y señale con un círculo el grado en que cada una de ellas se aplica a su matrimonio. Conteste lo más sinceramente posible.

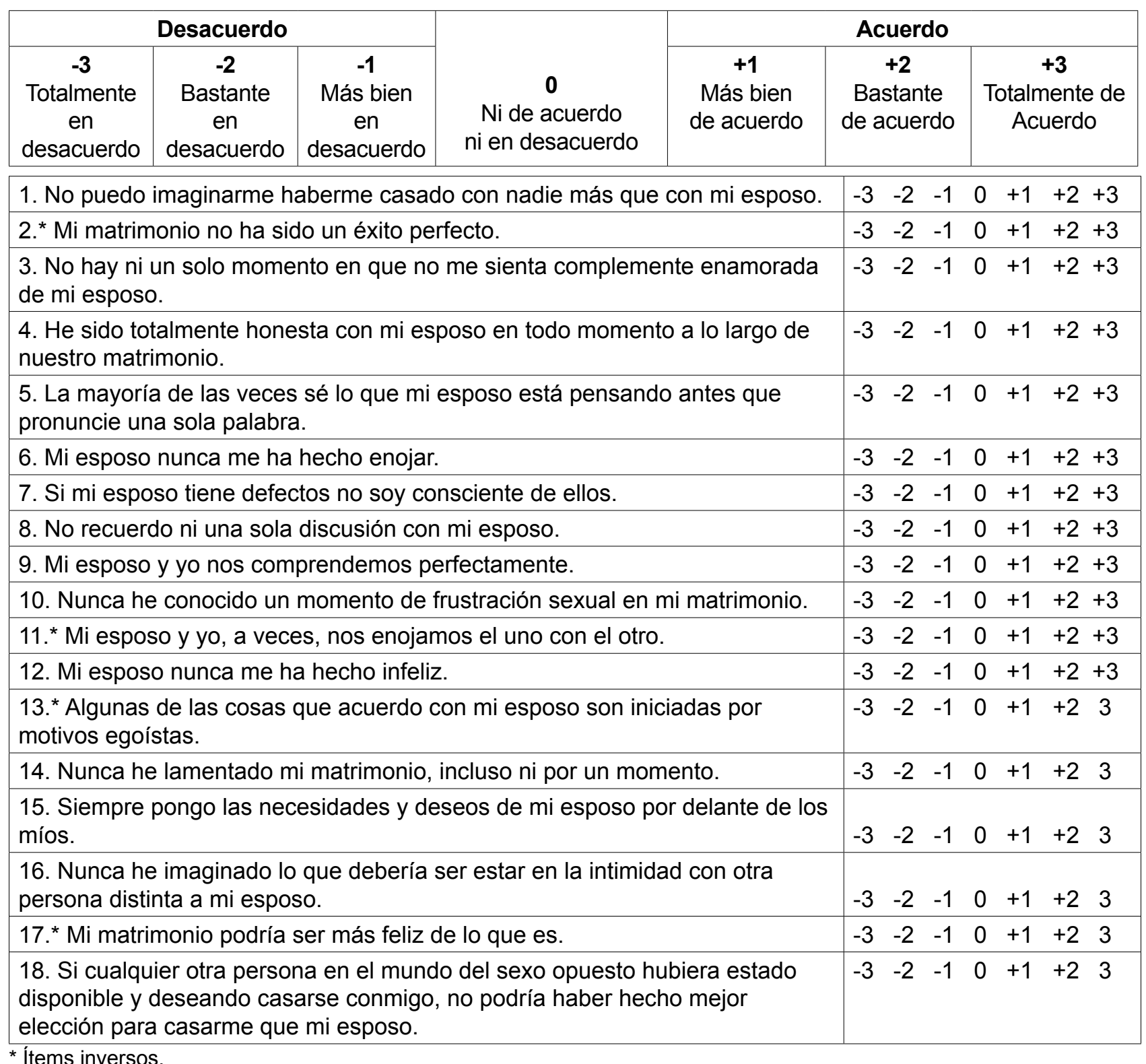

